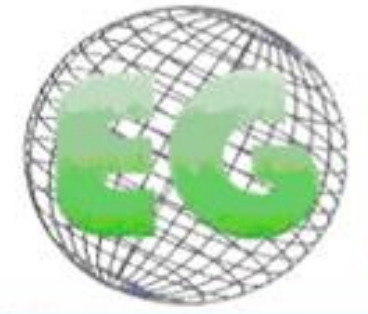

$N^{\circ} 39$

www.um.es/egloball

\title{
REVISIONES
}

\section{Instrumentos para la evaluación de la atención primaria de salud: una revisión narrativa}

Instruments for primary health care evaluation: a narrative review

\section{*Vélez Álvarez, Consuelo **Giraldo Osorio, Alexandra}

\author{
*Departamento de Salud Pública, Grupo de Investigación Promoción de la Salud y Prevención de la \\ Enfermedad, Universidad de Caldas. E-mail: consuelo.velez@ucaldas.edu.co **Grupo de \\ Investigación Promoción de la Salud y Prevención de la Enfermedad, Programa de Jóvenes
} Investigadores e Innovadores, Universidad de Caldas, Colciencias. Colombia.

Palabras clave: Atención primaria de salud; evaluación; validez de las pruebas; cuestionarios; estudios de validación; evaluación en salud; revisión

Keywords: primary health care; evaluation; validity of tests; questionnaires; validation studies; health evaluation; review

\section{RESUMEN}

Objetivo: Realizar una revisión narrativa sobre artículos que presentan instrumentos utilizados para la evaluación de la atención primaria de salud a nivel mundial.

Materiales y métodos: La búsqueda se realizó en Pubmed, Embase, Cochrane y Lilacs de artículos publicados entre 2008 y 2013. Los descriptores utilizados fueron: primary health care, health care evaluation mechanisms, questionnaires and validation study. Como criterios de elegibilidad de los artículos se incluyeron tres idiomas y estudios de validación. Se construyó una matriz de análisis por base de datos con las características de cada artículo, luego se consolidó una matriz final con los artículos que cumplieron con los criterios de inclusión y se analizaron los instrumentos encontrados. El análisis de la información se llevó a cabo por las dos autoras de forma independiente, para una mayor objetividad.

Resultados: Se obtuvieron 128 artículos que cumplieron con los criterios de inclusión establecidos en el estudio. El porcentaje de instrumentos identificados por categorías de análisis fueron: Estilos de vida y enfermedades crónicas 20,3\%, Estrategia de APS - Administración 5,4\%, Estrategia de APS Relación Equipo de salud Usuario 23,4\%, Salud Mental 28,9\% y otras áreas temáticas 21,9\%.

Conclusiones: Existe una amplia y variada disponibilidad de instrumentos genéricos y específicos que han sido validados en el contexto mundial para evaluar la estrategia de atención primaria de salud, sobre los cuales es posible reconstruir la mejor evidencia como herramienta de trabajo. 


\section{ABSTRACT}

Objective: To conduct a narrative review about articles that present instruments used for primary health care evaluation worldwide.

Materials and methods: The search was performed in PubMed, Embase, Cochrane, and Lilacs of articles published between 2008 and 2013. The descriptors used were: primary health care, health care evaluation mechanisms, questionnaires and validation study. The eligibility criteria for articles were three languages and validation studies. An analysis matrix was constructed by database with the characteristics of each item, then a final matrix was consolidated with the articles that met the inclusion criteria and the found instruments were analyzed. The data analysis was carried out by the two authors independently for greater objectivity.

Results: 128 articles that met the inclusion criteria for the study were obtained. The percentage of instruments identified by analysis categories were: Lifestyle and chronic diseases $20.3 \%$, PHC Management 5.4\% PHC - Relationship between Health equipment and User $23.4 \%$, Mental health 28, $9 \%$ and other subject areas $21.9 \%$.

Conclusions: There is a wide and varied availability of generic and specific instruments that have been validated in the global context for evaluating the primary health care strategy, from which it is possible to reconstruct the best evidence as a working tool.

\section{INTRODUCCIÓN}

La reorientación de los servicios de salud después de la renovación de la atención primaria de salud (APS) en las Américas, la ha sugerido como eje central de los sistemas de salud ${ }^{(1-2)}$. Esto ha llevado a la inclusión de mecanismos que permitan apropiaciones conceptuales, organizativas y de oferta de servicios en diferentes países de América Latina, ya que cada uno deberá encontrar su propio camino para crear una estrategia sostenible con el fin de basar sólidamente su sistema de salud en APS $^{(1)}$. Al respecto, uno de los principales retos derivados de la implementación de los sistemas de salud basados en APS, es la evaluación de los resultados de las actuaciones en términos de eficiencia y de cambios en la situación de salud de la población, con el fin de asegurar la calidad de los servicios ${ }^{(3-4)}$. De esta manera, la evaluación se constituye como un componente fundamental de la gestión y potencial fuente de insumos para la mejora de los procesos en general y la calidad en particular ${ }^{(3,5)}$.

La literatura ha reportado que los modelos teóricos sobre los que habitualmente se evalúa la APS enfatizan en aspectos estructurales y organizativos ${ }^{(6-8)}$ y escasamente el grado en que cumplen sus funciones ${ }^{(9)}$ y en este sentido, el volumen de estudios que se publican en revistas científicas crece enormemente, sin embargo, la calidad científica de los artículos de investigación es muy variada. La importancia de tener en cuenta la calidad de los estudios para la toma de decisiones, radica en que actuar con base a pruebas científicas inválidas o incorrectas es probablemente peor que actuar sin pruebas. En el mejor de los casos, la práctica se distorsiona de manera inconsciente y en el peor de ellos la decisión tomada puede causar daño a los pacientes 0 afectar negativamente a los usuarios del sistema de salud ${ }^{(10-11)}$. En el área de ciencias biomédicas y de salud se han realizado estudios con repercusión social pero pocos centrados en APS y aunque existen estudios enfocados en el análisis bibliométrico se requiere mayor actualización del nivel de producción científica en APS $^{(12)}$.

La evidencia científica reconstruida en esta revisión permitirá a los tomadores de decisiones e investigadores tener una visión integral sobre los instrumentos que han 
sido utilizados en diferentes contextos, áreas temáticas y líneas prioritarias objeto de estudio de la APS y de este manera identificar la información de los instrumentos para su fácil localización, consulta y selección. Es así, como se plantea realizar una revisión narrativa sobre artículos que presenten instrumentos utilizados para la evaluación de la APS a nivel mundial.

\section{MATERIALES Y MÉTODOS}

Los artículos de revisión narrativa son publicaciones amplias, apropiadas para describir y discutir el desarrollo o el "estado del arte" de un determinado asunto, bajo el punto de vista teórico o contextual ${ }^{(13)}$. Con este fin, para la búsqueda de la literatura de instrumentos que evalúan la APS se utilizaron 4 bases de datos electrónicas: Pubmed, Embase, Cochrane y Lilacs; se incluyeron artículos publicados entre los años 2008 y 2013. Además de las bases de datos se realizo búsqueda de referencias cruzadas de forma manual y literatura en revistas especializadas, en las cuales se encontraron duplicados algunos de los artículos ya seleccionados. Los términos Mesh, Decs y Emtree de búsqueda fueron: en atención primaria de salud (EJ, Primary health care), por la evaluación (EJ, health care evaluation mechanisms and questionnaires) y con el tipo de estudio (EJ, validation studies). Se construyó una matriz de análisis donde se registraron las características de cada uno de los artículos y se incluyeron variables como autores, título, idioma original, revista, año de publicación, objetivo, nombre del instrumento y dimensión evaluada; como resultado final de este proceso se obtuvieron cuatro matrices (una por cada base de datos). Como criterios de elegibilidad se establecieron artículos de validación de instrumentos para la evaluación de la APS en español, portugués e inglés y que los estudios presentaran un instrumento para evaluar la APS.

\section{Procesamiento de la Información}

La extracción de los datos de la revisión de los estudios se realizó con la participación de las dos investigadoras quienes trabajaron de manera independiente, revisaron los estudios y extrajeron los datos requeridos para diligenciar las matrices definidas para el análisis; el tipo de estudio y el instrumento utilizado para evaluar la APS. Posteriormente, se compararon los resultados de ambas y en caso de discrepancias, estas fueron resueltas por consenso. De las cuatro bases de datos se desarrolló una segunda matriz de artículos que cumplieran con los criterios de inclusión. Se revisaron los artículos publicados hasta junio del 2013 que fue el punto de corte para iniciar el análisis.

\section{Plan de análisis}

A partir de los datos consignados en la matriz final de consolidación se obtuvieron los artículos en texto completo, se analizaron las variables incluidas en este estudio y se realizó una categorización según el objetivo de evaluación propuesto por los instrumentos identificados (actores, programas y áreas temáticas a las que estaban dirigidos).

\section{Aspectos éticos}

Esta revisión sigue los parámetros éticos establecidos según la Resolución 8430 de 1993 y la Declaración de Helsinki y no evidencia riesgo para los humanos porque no se realiza sobre individuos sino sobre artículos publicados. 


\section{RESULTADOS}

Se realizó la búsqueda de los diferentes artículos en la bases de datos Pubmed, EMBASE, Cochrane y Lilacs, para lo cual se utilizaron diferentes estrategias de búsqueda las cuales se describen en la Tabla I.

\section{Tabla I: Historial y estrategias de búsqueda}

\begin{tabular}{|c|c|}
\hline $\begin{array}{c}\text { Base de } \\
\text { datos }\end{array}$ & Procedimiento y términos de búsqueda \\
\hline Pubmed & $\begin{array}{l}\text { Límites: } \\
\quad \text { Humanos } \\
2008 \text { a } 2013 \\
\text { \#1 Search "Primary Health Care"[Mesh] Filters: Publication date from 2008/01/01 to 2013/12/31; } \\
\text { Humans } \\
\text { \#2 Search Health Care Evaluation Mechanisms Filters: Publication date from 2008/01/01 to } \\
\text { 2013/12/31; Humans } \\
\text { \#3 Search "Questionnaires"[Mesh] Filters: Publication date from 2008/01/01 to 2013/12/31; Humans } \\
\text { \#4 Search "Validation Studies" [Publication Type] Filters: Publication date from 2008/01/01 to } \\
\text { 2013/12/31; Humans } \\
\text { \#5 Search (((\#1) AND \#2) AND \#3) AND \#4 }\end{array}$ \\
\hline Embase & $\begin{array}{l}\text { Límites: } \\
\text { Humanos } \\
\text { 2008 a } 2013 \\
\text { \#1. 'primary health care'/exp } \\
\text { \#2. 'primary health care'/exp AND (2008:py OR 2009:py OR 2010:py OR 2011:py OR 2012:py OR } \\
\text { 2013:py) } \\
\text { \#3. 'primary health care'/exp AND (2008:py OR 2009:py OR 2010:py OR 2011:py OR 2012:py OR } \\
\text { 2013:py) AND 'human'/de } \\
\text { \#4. 'health care quality'/exp } \\
\text { \#5. 'health care quality'/exp AND 'human'/de AND (2008:py OR 2009:py OR 2010:py OR 2011:py OR } \\
\text { 2012:py OR 2013:py OR 2014:py) } \\
\text { \#6. 'questionnaire'/exp } \\
\text { \#7. 'questionnaire'/exp AND 'human'/de AND (2008:py OR 2009:py OR 2010:py OR 2011:py OR } \\
\text { 2012:py OR 2013:py OR 2014:py) } \\
\text { \#8. 'primary health care'/exp AND (2008:py OR 2009:py OR 2010:py OR 2011:py OR 2012:py OR } \\
\text { 2013:py) AND 'health care quality'/exp AND 'questionnaire'/exp AND 'human'/de AND (2008:py OR } \\
\text { 2009:py OR 2010:py OR 2011:py OR 2012:py OR 2013:py OR 2014:py) } \\
\text { \#9. 'validation study'/exp } \\
\text { \#10. 'validation study'/exp AND 'human'/de AND (2008:py OR 2009:py OR 2010:py OR 2011:py OR } \\
\text { 2012:py OR 2013:py) } \\
\text { \#11. 'primary health care'/exp AND 'health care quality'/exp AND 'questionnaire'/exp AND (2008:py } \\
\text { OR 2009:py OR 2010:py OR 2011:py OR 2012:py OR 2013:py OR 2014:py) AND 'validation } \\
\text { study'/exp AND 'human'/de AND (2008:py OR 2009:py OR 2010:py OR 2011:py OR 2012:py OR } \\
\text { 2013:py) }\end{array}$ \\
\hline $\begin{array}{l}\text { Biblioteca } \\
\text { Cochrane }\end{array}$ & $\begin{array}{l}\text { \#1 primary health care } \\
\# 2 \text { health Care Evaluation Mechanisms } \\
\# 3 \text { questionnaires } \\
\# 4 \text { validation studies } \\
\# 5 \text { (\#1 and \#2 and \#3 and \#4) }\end{array}$ \\
\hline Lilacs & $\begin{array}{l}\text { Límites: } \\
\text { Humanos } \\
2008 \text { a } 2013 \\
\text { \#1 primary health care } \\
\text { \#2 health Care Evaluation Mechanisms } \\
\text { \#3 questionnaires } \\
\text { \#5 (\#1 and \#2 and \#3) }\end{array}$ \\
\hline
\end{tabular}

Fuente: elaboración propia

En la búsqueda especifica en cada base de datos se utilizaron los términos primary health care and heatlh care evaluation and questionnaires, excepto en la base de datos de Pubmed, en donde en lugar de utilizar el término heatlh care evaluation, se utilizó heatlh care quality. Como resultados de esta primera búsqueda obtuvieron 4141 artículos. Posteriormente se afinó la búsqueda y se seleccionaron estudios de validación, por lo que se restringieron los resultados a 222 de los cuales en total 128 
cumplieron con los criterios de inclusión y fueron anexados a la matriz de los evaluadores (Tabla II). Finalmente fueron rechazados 78 artículos por no cumplir con los criterios de inclusión y 16 por estar duplicados en las diferentes bases de datos. Posterior a la evaluación de los instrumentos encontrados, se realizó una categorización teniendo en cuenta los que evaluaban las funciones de la APS de manera directa, los que evaluaban las funciones de la APS en relación con un área temática, y los que evaluaban áreas temáticas especificas sin circunscribirse a ninguna de las funciones de la APS. Tras el análisis las categorías que emergieron fueron: Estilos de vida y enfermedades crónicas (20,3\%), Estrategia de APS Administración (5,4\%), Estrategia de APS - Relación Equipo de salud Usuario $(23,4 \%)$, Salud Mental $(28,9 \%)$ y otras áreas temáticas $(21,9 \%)$. En esta última categoría, se incluyeron instrumentos dirigidos a evaluar las áreas temáticas relacionadas con la actividad física, los sistemas musculo esquelético, digestivo y nervioso; salud ocupacional, salud general, salud sexual y reproductiva y el cuidado de la salud del adulto mayor.

\section{Tabla II: Resultados finales de la búsqueda de artículos}

\begin{tabular}{|l|c|c|c|c|}
\hline \multicolumn{1}{|c|}{$\begin{array}{c}\text { Base } \\
\text { datos }\end{array}$} & $\begin{array}{c}\text { Estrategia } \\
\text { de búsqueda }\end{array}$ & $\begin{array}{c}\text { No. artículos } \\
\text { encontrados }\end{array}$ & $\begin{array}{c}\text { No. artículos } \\
\text { seleccionados por } \\
\text { corresponder a } \\
\text { estudios de validación }\end{array}$ & $\begin{array}{c}\text { No. artículos que } \\
\text { cumplieron con los } \\
\text { criterios de } \\
\text { inclusión }\end{array}$ \\
\hline Pubmed & $\begin{array}{c}\text { primary health care and heatlh } \\
\text { care evaluation and } \\
\text { questionnaires }\end{array}$ & 2638 & 146 & 118 \\
\hline Embase & $\begin{array}{c}\text { primary health care and heatlh } \\
\text { care quality and questionnaires }\end{array}$ & 1456 & 29 & 10 \\
\hline Cochrane & $\begin{array}{c}\text { primary health care and heatlh } \\
\text { care quality and questionnaires }\end{array}$ & 39 & 39 & 0 \\
\hline Lilacs & $\begin{array}{c}\text { primary health care and heatlh } \\
\text { care quality and questionnaires }\end{array}$ & 8 & 8 & 0 \\
\hline Total & & 4141 & 222 & 128 \\
\hline
\end{tabular}

Fuente: elaboración propia

En las Tablas III a la VII, se presentan los instrumentos encontrados por cada una de las categorías que emergieron. Al analizar las aéreas de evaluación nótese como en la categoría estilos de vida y enfermedades crónicas la tendencia es que para evaluar conductas (aspectos psicosociales y personales) se encontraron 14 de los 26 instrumentos (53\%). Dentro de los instrumentos para evaluar la estrategia de APS se encontraron 37 de los cuales en el área temática de administración se encontraron $7(19 \%)$ y $30(81 \%)$ para evaluar la relación equipo de salud-usuario. Coherente con el desarrollo que ha tenido la APS a nivel mundial se han diseñado gran cantidad de instrumentos para evaluar la salud mental (37) y en otras áreas temáticas se encontraron 28 instrumentos. La Gráfica 1 muestra la tendencia de los instrumentos en cada temática por año. 


\section{Gráfica 1: Tendencias por año de las áreas que evalúan los instrumentos}

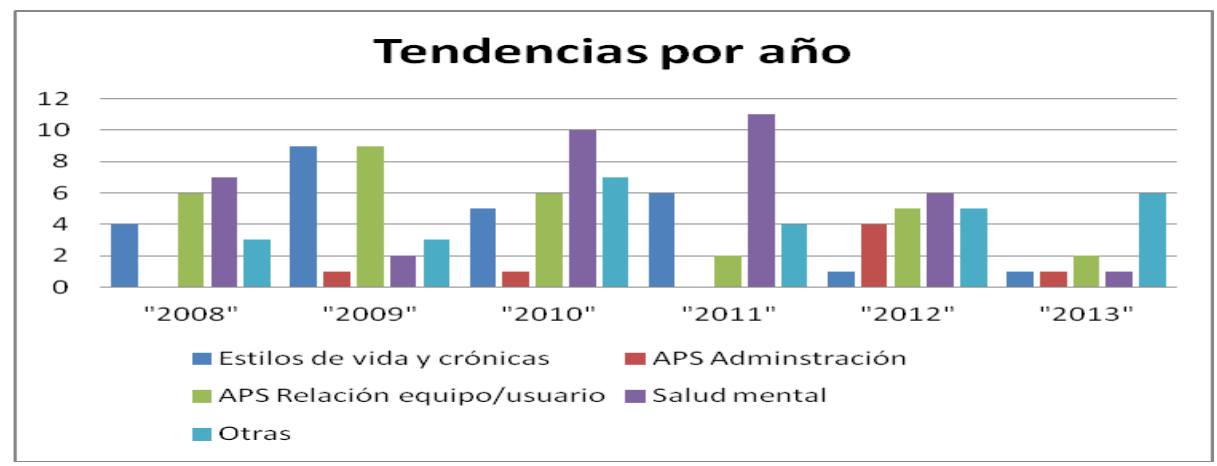

Fuente: elaboración propia

Tabla III: Instrumentos para evaluar estilos de vida y enfermedadescrónicas $n=26$

\begin{tabular}{|c|c|c|c|}
\hline Autores & Año & Instrumento & $\begin{array}{l}\text { Área de evaluación- Objetivo del } \\
\text { instrumento }\end{array}$ \\
\hline $\begin{array}{l}\text { De Torres LA, Rebollo } \\
\text { EM, Ruiz-Moral R, et } \\
\mathrm{al}^{+}\end{array}$ & 2009 & $\begin{array}{l}\text { AUDIT (Alcohol Use Disorders } \\
\text { Identification Test) }\end{array}$ & $\begin{array}{l}\text { Identificar trastornos por alcohol } \\
\text { (Conducta) }\end{array}$ \\
\hline $\begin{array}{l}\text { Seid M, Opipari- } \\
\text { Arrigan L, et al }^{+}\end{array}$ & 2009 & $\mathrm{BCQ}$ & Evaluar el asma en niños (Clínica) \\
\hline $\begin{array}{l}\text { Guck TP, Banfield MA, } \\
\text { Tran SM, et al }{ }^{+}\end{array}$ & 2008 & $\begin{array}{l}\text { Multidimensional Diabetes } \\
\text { Questionnaire and the Brief } \\
\text { Symptom Inventory (BSI) }\end{array}$ & $\begin{array}{l}\text { Aspectos psicosociales en pacientes con } \\
\text { diabetes (Conducta) }\end{array}$ \\
\hline $\begin{array}{l}\text { Dunderdale } \mathrm{K}, \\
\text { Thompson DR, et al }^{+}\end{array}$ & 2008 & CHAT & $\begin{array}{l}\text { Calidad de vida de los pacientes } \\
\text { (Conducta) }\end{array}$ \\
\hline $\begin{array}{l}\text { Ställberg B, Nokela M, } \\
\text { Ehrs PO, et al }\end{array}$ & 2009 & Clinical COPD Questionnaire & Evaluar EPOC (Clínica) \\
\hline $\begin{array}{l}\text { Lennon OC, Carey A, } \\
\text { Creed A, et } \mathrm{al}^{+}\end{array}$ & 2011 & $\begin{array}{l}\text { COOP/WONCA charts for } \\
\text { community-dwelling stroke } \\
\text { survivors }\end{array}$ & $\begin{array}{l}\text { Evaluar sobrevivientes de la enfermedad } \\
\text { cerebro vascular (Clínica) }\end{array}$ \\
\hline John IA, Lawoko S ${ }^{+}$ & 2010 & DVHPSS & $\begin{array}{l}\text { Identificar la violencia en pareja } \\
\text { (conducta) }\end{array}$ \\
\hline $\begin{array}{l}\text { Frith } P, \text { Crockett A, } \\
\text { Beilby J, et al }\end{array}$ & 2011 & $\begin{array}{l}\text { FEV1/FEV6 (Forced expiratory } \\
\text { volume ratio at one and six } \\
\text { seconds) }\end{array}$ & Detectar EPOC (Clínica) \\
\hline $\begin{array}{l}\text { Van Dijk-de Vries AN, } \\
\text { Duimel-Peeters IG, } \\
\text { Vrijhoef } \mathrm{HJ}^{+}\end{array}$ & 2011 & $\begin{array}{l}\text { HEPRODIA (Health Promotion } \\
\text { Diabetes) }\end{array}$ & Diabetes (Conducta) \\
\hline $\begin{array}{l}\text { Karademir M, } \\
\text { Koseoglu IH, } \\
\text { Vatansever K, el } \mathrm{al}^{+}\end{array}$ & 2009 & $\begin{array}{l}\text { Hill-Bone compliance to high } \\
\text { blood pressure therapy scale }\end{array}$ & $\begin{array}{l}\text { Tratamiento de la hipertensión arterial } \\
\text { (Clínica) }\end{array}$ \\
\hline Day LA, Brice $\mathrm{P}^{+}$ & 2013 & $\begin{array}{l}\text { HPP/HPQ (The Hearing Parents } \\
\text { Perceptions of Health } \\
\text { Professionals Advice } \\
\text { Questionnaire) }\end{array}$ & $\begin{array}{l}\text { Percepciones de los padres frente a los } \\
\text { consejos de los profesionales de salud } \\
\text { (Conducta) }\end{array}$ \\
\hline $\begin{array}{l}\text { Garcia-Esteve L, } \\
\text { Torres A, Navarro P, et } \\
\mathrm{al}^{+}\end{array}$ & 2011 & $\begin{array}{l}\text { ISA (Index of Spouse Abuse) } \\
\text { PMWI-SF (Psychological } \\
\text { Maltreatment of Women } \\
\text { Inventory short form) } \\
\text { WAST (Woman Abuse } \\
\text { Screening Tool) } \\
\text { PVS (Partner Violence Screen) }\end{array}$ & $\begin{array}{l}\text { Identificar la violencia doméstica en } \\
\text { centros de salud (Conducta) }\end{array}$ \\
\hline $\begin{array}{l}\text { Van de Steeg N, Sielk } \\
\text { M, Pentzek M, et al }{ }^{+}\end{array}$ & 2009 & $\begin{array}{l}\text { MARS-5 (Morisky and } \\
\text { Medication Adherence Report } \\
\text { Scale) }\end{array}$ & $\begin{array}{l}\text { Adherencia de los pacientes tratados con } \\
\text { fármacos antihipertensivos en APS } \\
\text { (Conducta) }\end{array}$ \\
\hline $\begin{array}{l}\text { Naveiro-Rilo JC, Diez- } \\
\text { Juárez DM, Romero } \\
\text { Blanco A, el al }{ }^{+}\end{array}$ & 2010 & MLHFQ & Insuficiencia cardíaca (clínica) \\
\hline $\begin{array}{l}\text { Baena-Díez JM, } \\
\text { Alzamora-Sas MT, } \\
\text { Grau M, et al }{ }^{+}\end{array}$ & 2009 & $\begin{array}{l}\text { MONICA cardiovascular } \\
\text { questionnaire }\end{array}$ & $\begin{array}{l}\text { Determinantes de las enfermedades } \\
\text { cardiovasculares en comparación con la } \\
\text { historia clínica (Clínica) }\end{array}$ \\
\hline
\end{tabular}




\begin{tabular}{|c|c|c|c|}
\hline $\begin{array}{l}\text { Cramm JM, Nieboer } \\
\mathrm{AP}^{+}\end{array}$ & 2012 & $\begin{array}{l}\text { PACIC (Patient Assessment of } \\
\text { Chronic Illness Care) } \\
\text { PACIC-S (PACIC short version) }\end{array}$ & $\begin{array}{l}\text { Atención de las enfermedad crónicas } \\
\text { (Conducta) }\end{array}$ \\
\hline $\begin{array}{l}\text { Ballal MS, } \\
\text { Selvachandran SN, } \\
\text { Maw A }\end{array}$ & 2010 & $\begin{array}{l}\text { PCQ (Patient Consultation } \\
\text { Questionnaire) }\end{array}$ & Detección del cáncer colorrectal (Cínica) \\
\hline $\begin{array}{l}\text { Bowling } A \text {, Reeves } B \text {, } \\
\text { Rowe } \mathrm{G}^{+}\end{array}$ & 2010 & $\begin{array}{l}\text { PPQA (Patient Preferences } \\
\text { Questionnaire for Angina } \\
\text { treatment) }\end{array}$ & Tratamiento de angina (clínica) \\
\hline $\begin{array}{l}\text { Coulibaly A, Turgeon } \\
\text { O'Brien H, Galibois I }^{+}\end{array}$ & 2009 & QFFQ & $\begin{array}{l}\text { Evaluar la ingesta de proteínas en la dieta } \\
\text { de pacientes con diabetes (Conducta) }\end{array}$ \\
\hline $\begin{array}{l}\text { Wei X, Barnsley J, } \\
\text { Zakus D, et al }^{+}\end{array}$ & 2008 & $\begin{array}{l}\text { Questionnaire based on agency } \\
\text { theory }\end{array}$ & Diabetes (clínica) \\
\hline $\begin{array}{l}\text { Borschmann RD, } \\
\text { Cottrell } \mathrm{D}^{+}\end{array}$ & 2009 & $\begin{array}{l}\text { RASP-B (Readiness to alter } \\
\text { sun-protective behaviour } \\
\text { questionnaire) }\end{array}$ & $\begin{array}{l}\text { Cambio de comportamiento para } \\
\text { exposición al sol (Conducta) }\end{array}$ \\
\hline $\begin{array}{l}\text { Sigel E, Hart J, } \\
\text { Hoffenberg A, Dodge } \\
\mathrm{M}^{+}\end{array}$ & 2011 & Screening tool & $\begin{array}{l}\text { Detección de jóvenes de violencia futura } \\
\text { (Conducta) }\end{array}$ \\
\hline $\begin{array}{l}\text { Ashton-Prolla P, } \\
\text { Giacomazzi J, et al }\end{array}$ & 2009 & Simple questionnaire & $\begin{array}{l}\text { Identificación de cáncer de mama } \\
\text { hereditario en APS (clínica) }\end{array}$ \\
\hline 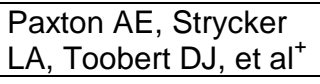 & 2011 & STC tool & $\begin{array}{l}\text { Instrumento para la evaluación y el } \\
\text { asesoramiento de la dieta (Conducta) }\end{array}$ \\
\hline $\begin{array}{l}\text { Rebollo P, Cuervo J, } \\
\text { Villa G, et } \mathrm{al}^{+}\end{array}$ & 2010 & The SAT-Q Questionnaire & $\begin{array}{l}\text { Evaluación de la satisfacción de los } \\
\text { pacientes con enfermedad crónica } \\
\text { (Conducta) }\end{array}$ \\
\hline $\begin{array}{l}\text { Plazaola-Castaño J, } \\
\text { Ruiz-Pérez I, } \\
\text { Hernández-Torres } \mathrm{E}^{+}\end{array}$ & 2008 & Woman Abuse Screening Tool & $\begin{array}{l}\text { Evaluar mujeres maltratadas entre las } \\
\text { asistentes a los servicios de APS } \\
\text { (Conducta) }\end{array}$ \\
\hline
\end{tabular}

${ }^{+}$Base de datos de la que se obtuvo el estudio: Pubmed

\section{Tabla IV: Instrumentos para evaluar la estrategia de APS - Administración $\mathbf{n = 7}$}

\begin{tabular}{|l|l|l|l|}
\hline \multicolumn{1}{|c|}{ Autores } & Año & \multicolumn{1}{|c|}{ Instrumento } & \multicolumn{1}{|c|}{$\begin{array}{c}\text { Área de evaluación- Objetivo del } \\
\text { Instrumento }\end{array}$} \\
\hline $\begin{array}{l}\text { Pezzolesi C, Manser T, } \\
\text { Schifano F, et al }\end{array}$ & 2013 & $\begin{array}{l}\text { HPT (Handover Performance } \\
\text { Tool) }\end{array}$ & $\begin{array}{l}\text { Evaluar sistemáticamente la calidad } \\
\text { y seguridad de los cambios de turno }\end{array}$ \\
\hline $\begin{array}{l}\text { Bobiak SN, Zyzanski SJ, } \\
\text { Ruhe MC, et al }\end{array}$ & 2009 & $\begin{array}{l}\text { Measuring practice capacity for } \\
\text { change }\end{array}$ & Evaluar la capacidad de cambio \\
\hline $\begin{array}{l}\text { Benachi Sandoval N, } \\
\text { Castillo Martínez A, } \\
\text { Vilaseca Llobet JM, et al }{ }^{+}\end{array}$ & 2012 & $\begin{array}{l}\text { PCAS (Primary Care Assessment } \\
\text { Survey) }\end{array}$ & $\begin{array}{l}\text { Evaluar fortalezas y debilidades de la } \\
\text { APS }\end{array}$ \\
\hline $\begin{array}{l}\text { Kennie-Kaulbach N, Farrell } \\
\text { B, et al }{ }^{+}\end{array}$ & 2012 & Pharmacists' competencies & $\begin{array}{l}\text { Evaluar el desempeño de los } \\
\text { farmacéuticos en APS }\end{array}$ \\
\hline $\begin{array}{l}\text { Ose D, Freund T, Kunz CU, } \\
\text { et al }{ }^{+}\end{array}$ & 2010 & $\begin{array}{l}\text { SOAPC (communication, } \\
\text { decision making, stress/chaos, } \\
\text { history of change) }\end{array}$ & $\begin{array}{l}\text { Evaluar los atributos } \\
\text { organizacionales }\end{array}$ \\
\hline $\begin{array}{l}\text { Wholey DR, Zhu X, Knoke } \\
\text { D, et al }{ }^{+}\end{array}$ & 2012 & $\begin{array}{l}\text { TACT (The Teamwork in } \\
\text { assertive community treatment) }\end{array}$ & $\begin{array}{l}\text { Evaluar el papel de los procesos de } \\
\text { equipo en el desempeño del } \\
\text { tratamiento comunitario asertivo }\end{array}$ \\
\hline $\begin{array}{l}\text { Haller DM, Meynard A, } \\
\text { Pejic D, et al }{ }^{+}\end{array}$ & 2012 & YFHS-WHO+ Questionnaire & $\begin{array}{l}\text { Evaluar el acceso a los servicios de } \\
\text { APS por parte de los jóvenes }\end{array}$ \\
\hline
\end{tabular}

${ }^{+}$Base de datos de la que se obtuvo el estudio: Pubmed

\section{Tabla V: Instrumentos para evaluar la estrategia de APS - Relación Equipo de} salud Usuario $n=30$

\begin{tabular}{|l|c|l|l|}
\hline \multicolumn{1}{|c|}{ Autores } & Año & \multicolumn{1}{c|}{ Instrumento } & $\begin{array}{c}\text { Área de evaluación - Objetivo } \\
\text { del Instrumento }\end{array}$ \\
\hline $\begin{array}{l}\text { Hadjistavropoulos H, Biem H, } \\
\text { et } \mathrm{al}^{+}\end{array}$ & 2008 & Care Questionnaire & $\begin{array}{l}\text { Evaluar la continuidad de la } \\
\text { atención del paciente }\end{array}$ \\
\hline $\begin{array}{l}\text { Letelier MJ, Aller MB, Henao } \\
\mathrm{D}, \mathrm{et} \mathrm{al}^{+}\end{array}$ & 2010 & $\begin{array}{l}\text { CCAENA (Continuidad asistencial } \\
\text { entre niveles desde la perspectiva } \\
\text { del usuario) }\end{array}$ & $\begin{array}{l}\text { Evaluar la perspectiva de los } \\
\text { usuarios frente a continuidad en } \\
\text { los diferentes niveles de } \\
\text { atención }\end{array}$ \\
\hline
\end{tabular}




\begin{tabular}{|c|c|c|c|}
\hline $\begin{array}{l}\text { Gavilán Moral E, Ruiz Moral } \\
\text { R, et } \mathrm{al}^{+}\end{array}$ & 2010 & CICAA scale & $\begin{array}{l}\text { Evaluar la atención centrada en } \\
\text { el paciente }\end{array}$ \\
\hline $\begin{array}{l}\text { Ruiz Moral R, Peralta } \\
\text { Munguia L, Pérula de Torres } \\
\text { LA, et al }{ }^{+}\end{array}$ & 2010 & $\begin{array}{l}\text { CICAA-Decision (General } \\
\text { communication skills) }\end{array}$ & $\begin{array}{l}\text { Incluir a los pacientes en las } \\
\text { decisiones en APS }\end{array}$ \\
\hline $\begin{array}{l}\text { Berendsen AJ, Groenier } \mathrm{KH} \text {, } \\
\text { De Jong GM, et al }{ }^{+}\end{array}$ & 2009 & CQ-index (Consumer Quality Index) & $\begin{array}{l}\text { Evaluar el personal médico y } \\
\text { especialistas por los pacientes }\end{array}$ \\
\hline $\begin{array}{l}\text { Bakshi AB, Wee SL, Tay C, } \\
\text { et al }\end{array}$ & 2012 & CTM-15 and CTM-3 & $\begin{array}{l}\text { Evaluar la transición del cuidado } \\
\text { por el paciente }\end{array}$ \\
\hline $\begin{array}{l}\text { Edvardsson D, Koch S, Nay } \\
\mathrm{R}^{+}\end{array}$ & 2009 & $\begin{array}{l}\text { English Language Person-Centered } \\
\text { Climate Questionnaire-Patient } \\
\text { version }\end{array}$ & $\begin{array}{l}\text { Evaluar el clima del centro de } \\
\text { salud centrado en el paciente }\end{array}$ \\
\hline $\begin{array}{l}\text { Haggerty } \mathrm{JL} \text {, Roberge } \mathrm{D} \text {, } \\
\text { Freeman } \mathrm{GK} \text {, et } \mathrm{al}^{+}\end{array}$ & 2012 & $\begin{array}{l}\text { Generic Measure of Continuity of } \\
\text { Care }\end{array}$ & $\begin{array}{l}\text { Evaluar la continuidad de la } \\
\text { atención desde la perspectiva } \\
\text { del paciente }\end{array}$ \\
\hline Mead N, Bower P, Roland $\mathrm{M}^{+}$ & 2008 & $\begin{array}{l}\text { GPAQ (General Practice } \\
\text { Assessment Questionnaire) }\end{array}$ & $\begin{array}{l}\text { Evaluar la calidad de la APS, } \\
\text { acceso y atención interpersonal }\end{array}$ \\
\hline $\begin{array}{l}\text { Gremigni } \mathrm{P}, \text { Sommaruga } \mathrm{M} \text {, } \\
\text { Peltenburg } \mathrm{M}^{+}\end{array}$ & 2008 & $\begin{array}{l}\text { HCCQ (Health Care } \\
\text { Communication Questionnaire) }\end{array}$ & $\begin{array}{l}\text { Evaluar la comunicación } \\
\text { ambulatoria con personal no } \\
\text { médico del hospital }\end{array}$ \\
\hline $\begin{array}{l}\text { Suhonen R, Gustafsson ML, } \\
\text { et } \mathrm{al}^{+}\end{array}$ & 2010 & $\begin{array}{l}\text { ICS-Nurse (Individualized Care } \\
\text { Scale - Nurse) }\end{array}$ & $\begin{array}{l}\text { Evaluar el cuidado } \\
\text { individualizado en enfermería }\end{array}$ \\
\hline $\begin{array}{l}\text { Harley C, Adams J, Booth L, } \\
\text { et al }\end{array}$ & 2009 & MCQ (Medical Care Questionnaire) & $\begin{array}{l}\text { Evaluar pacientes ambulatorios } \\
\text { oncologicos }\end{array}$ \\
\hline $\begin{array}{l}\text { Graumlich JF, Grimmer- } \\
\text { Somers K, Aldag JC }{ }^{++}\end{array}$ & 2008 & $\begin{array}{l}\text { Modified Physician-PREPARED } \\
\text { scale }\end{array}$ & $\begin{array}{l}\text { Evaluar la percepción de la } \\
\text { calidad y el resultado de la } \\
\text { planificación del alta y la } \\
\text { comunicación por parte de los } \\
\text { médicos }\end{array}$ \\
\hline $\begin{array}{l}\text { Torijano-Casalengua ML, } \\
\text { Olivera-Cañadas G, Astier- } \\
\text { Peña MP, et al }{ }^{++} \\
\end{array}$ & 2013 & $\begin{array}{l}\text { MOSPSC (Medical Office Survey on } \\
\text { Patient Safety Culture) }\end{array}$ & $\begin{array}{l}\text { Evaluar la cultura de seguridad } \\
\text { del paciente }\end{array}$ \\
\hline $\begin{array}{l}\text { Vuković M, Gvozdenović BS, } \\
\text { Gajić T, et al }\end{array}$ & 2012 & Patient satisfaction questionnaire & $\begin{array}{l}\text { Evaluar la satisfacción de los } \\
\text { pacientes frente a los servicios } \\
\text { de medicina general }\end{array}$ \\
\hline $\begin{array}{l}\text { Carmen S, Teal S, Guzzetta } \\
\text { CE }^{+}\end{array}$ & 2008 & $\begin{array}{l}\text { Patient-Family-Centered Care } \\
\text { Survey }\end{array}$ & $\begin{array}{l}\text { Evaluar la atención al paciente } \\
\text { centrada en la familia }\end{array}$ \\
\hline $\begin{array}{l}\text { Pantoja T, Beltrán M, Moreno } \\
\mathrm{G}^{+}\end{array}$ & 2009 & $\begin{array}{l}\text { Patients' perspective in Chilean } \\
\text { primary care }\end{array}$ & $\begin{array}{l}\text { Evaluar la APS desde la } \\
\text { perspectiva de los pacientes }\end{array}$ \\
\hline $\begin{array}{l}\text { Lee JH, Choi YJ, Sung NJ, et } \\
\mathrm{al}^{+}\end{array}$ & 2009 & $\begin{array}{l}\text { PCAT (Primary care assessment } \\
\text { tool) }\end{array}$ & Evaluar el desempeño de la APS \\
\hline $\begin{array}{l}\text { Yang H, Shi L, Lebrun LA, et } \\
\mathrm{al}^{+}\end{array}$ & 2013 & $\begin{array}{l}\text { PCAT-C (Primary Care Assessment } \\
\text { Tool Chinese version) }\end{array}$ & $\begin{array}{l}\text { Calidad de la atención primaria } \\
\text { desde la perspectiva de los } \\
\text { usuarios }\end{array}$ \\
\hline $\begin{array}{l}\text { Edvardsson D, Koch S, Nay } \\
\mathrm{R}^{+}\end{array}$ & 2010 & $\begin{array}{l}\text { PCQ-S (English language Person- } \\
\text { centred Climate Questionnaire--staff } \\
\text { version) }\end{array}$ & $\begin{array}{l}\text { Evaluar el clima del centro de } \\
\text { salud como un aspecto centrado } \\
\text { en el personal de salud }\end{array}$ \\
\hline $\begin{array}{l}\text { Lam CL, Yuen NY, Mercer } \\
\text { SW, et } \mathrm{al}^{+}\end{array}$ & 2010 & r & $\begin{array}{l}\text { Evaluar la estrategia por } \\
\text { pacientes }\end{array}$ \\
\hline Dempster M, Donnelly $\mathrm{M}^{+}$ & 2008 & $\begin{array}{l}\text { Perceived Health Competence } \\
\text { Scale }\end{array}$ & $\begin{array}{l}\text { Salud percibida en el entorno de } \\
\text { APS }\end{array}$ \\
\hline $\begin{array}{l}\text { Edvardsson } \mathrm{D}, \text { Sandman } \mathrm{PO} \text {, } \\
\text { Rasmussen } \mathrm{B}^{+}\end{array}$ & 2009 & $\begin{array}{l}\text { Person-centred Climate } \\
\text { Questionnaire - staff version }\end{array}$ & $\begin{array}{l}\text { Evaluar la estrategia por } \\
\text { pacientes }\end{array}$ \\
\hline $\begin{array}{l}\text { Reinders ME, Blankenstein } \\
\mathrm{AH}, \mathrm{Knol} \mathrm{DL} \text {, et } \mathrm{al}^{+}\end{array}$ & 2009 & $\begin{array}{l}\text { PFC (Patient Feedback } \\
\text { Questionnaire on Consultation } \\
\text { Skills) }\end{array}$ & $\begin{array}{l}\text { Evaluar la satisfacción del } \\
\text { paciente frente a las habilidades } \\
\text { del médico }\end{array}$ \\
\hline 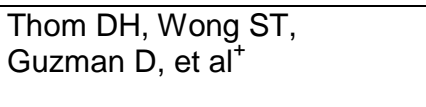 & 2011 & Physician Trust in the Patient & $\begin{array}{l}\text { Evaluar la confianza médico - } \\
\text { paciente }\end{array}$ \\
\hline $\begin{array}{l}\text { Shaw WS, Woiszwillo MJ, } \\
\text { Krupat } \mathrm{E}^{+}\end{array}$ & 2012 & $\begin{array}{l}\text { PPOS (Patient-Practitioner } \\
\text { Orientation Scale) }\end{array}$ & $\begin{array}{l}\text { Evaluar aspectos no biomédicos } \\
\text { relacionados con el dolor de } \\
\text { espalda }\end{array}$ \\
\hline $\begin{array}{l}\text { Puschel K, Repetto P, Solar } \\
M O \text {, et } \mathrm{al}^{+}\end{array}$ & 2012 & SALUFAM & $\begin{array}{l}\text { Evaluar familias de mayor } \\
\text { vulnerabilidad en salud }\end{array}$ \\
\hline $\begin{array}{l}\text { Lurie SJ, Schultz SH, } \\
\text { Lamanna G }^{+}\end{array}$ & 2011 & Teamwork-assessment & $\begin{array}{l}\text { Evaluar la función del equipo de } \\
\text { APS }\end{array}$ \\
\hline $\begin{array}{l}\text { Slater } \mathrm{P}, \text { McCormack B, } \\
\text { Bunting } \mathrm{B}^{+}\end{array}$ & 2009 & The Nursing Context Index & $\begin{array}{l}\text { Evaluar el cuidado de enfermería } \\
\text { centrado en la persona }\end{array}$ \\
\hline
\end{tabular}




\begin{tabular}{l}
\begin{tabular}{|l|l|l|}
\hline $\begin{array}{l}\text { Rose D, Sweeney A, Leese } \\
\text { M, et al }{ }^{+}\end{array} 2009$ & $\begin{array}{l}\text { User-generated measure of } \\
\text { continuity of care }\end{array}$ & $\begin{array}{l}\text { Evaluar la continuidad de la } \\
\text { atención }\end{array}$ \\
\hline
\end{tabular} \\
\hline
\end{tabular}

Base de datos de la que se obtuvo el estudio: Pubmed, Base de datos de la que se obtuvo el estudio:

Embase

Tabla VI: Instrumentos para evaluar la Salud Mental $n=\mathbf{3 7}$

\begin{tabular}{|c|c|c|c|}
\hline Autores & Año & Instrumento & $\begin{array}{c}\text { Área de evaluación - Objetivo } \\
\text { del Instrumento }\end{array}$ \\
\hline $\begin{array}{l}\text { Arroll } \mathrm{B} \text {, Fernando A, Falloon } \\
\mathrm{K} \text {, et } \mathrm{al}^{+}\end{array}$ & 2011 & Auckland Sleep Questionnaire & $\begin{array}{l}\text { Diagnosticar trastornos del sueño } \\
\text { en atención primaria }\end{array}$ \\
\hline $\begin{array}{l}\text { Caraveo-Anduaga JJ, } \\
\text { López-Jiménez JL, Soriano- } \\
\text { Rodríguez A, et } \mathrm{al}^{+}\end{array}$ & 2011 & CBTD & $\begin{array}{l}\text { Vigilancia de la salud mental en } \\
\text { niños y adolescentes }\end{array}$ \\
\hline $\begin{array}{l}\text { Vega WA, Rodriguez MA, } \\
\text { Ang } A^{+}\end{array}$ & 2010 & Checklist & Tratamiento de la depression \\
\hline $\begin{array}{l}\text { Cape J, Morris E, Burd M, } \\
\text { Buszewicz } \mathrm{M}^{++}\end{array}$ & 2008 & $\begin{array}{l}\text { Complexity of psychosocial } \\
\text { explanations measure }\end{array}$ & $\begin{array}{l}\text { Explicaciones de los problemas } \\
\text { comunes de salud mental }\end{array}$ \\
\hline Finnell DS, Lee $\mathrm{J}^{+}$ & 2011 & $\begin{array}{l}\text { Decisional Balance for Patient } \\
\text { Choice in Substance Abuse } \\
\text { Treatment }\end{array}$ & $\begin{array}{l}\text { Evaluar los pro y los contra de } \\
\text { elegir tratamiento para el abuso de } \\
\text { sustancias }\end{array}$ \\
\hline $\begin{array}{l}\text { Gloster AT, Rhoades HM, } \\
\text { Novy D, et } \mathrm{al}^{+}\end{array}$ & 2008 & $\begin{array}{l}\text { Depression Anxiety and Stress } \\
\text { Scale- } 21 \text { in older primary care } \\
\text { patients }\end{array}$ & $\begin{array}{l}\text { Evaluar depresión, ansiedad y } \\
\text { estrés en adultos mayores }\end{array}$ \\
\hline $\begin{array}{l}\text { Pietsch K, Allgaier AK, Frühe } \\
\mathrm{B} \text {, et al }\end{array}$ & 2011 & $\begin{array}{l}\text { DesTeen (Depression Screener for } \\
\text { Teenagers) }\end{array}$ & $\begin{array}{l}\text { Tamizaje de la depresión en } \\
\text { adolescentes }\end{array}$ \\
\hline $\begin{array}{l}\text { García-Campayo J, } \\
\text { Zamorano E, et al }^{+}\end{array}$ & 2012 & $\begin{array}{l}\text { GAD (Generalised Anxiety } \\
\text { Disorder) }\end{array}$ & $\begin{array}{l}\text { Detectar pacientes con probable } \\
\text { trastorno de ansiedad }\end{array}$ \\
\hline $\begin{array}{l}\text { Sidik SM, Arroll B, } \\
\text { Goodyear-Smith F }\end{array}$ & 2012 & GAD-7 & Detectar ansiedad en mujeres \\
\hline $\begin{array}{l}\text { Castelo MS, Coelho-Filho } \\
\mathrm{JM} \text {, et } \mathrm{al}^{+}\end{array}$ & 2010 & GDS (Geriatric Depression Scale) & Depresión geriatrica \\
\hline $\begin{array}{l}\text { Pirani A, Brodaty H, Martini } \\
\mathrm{E} \text {, et } \mathrm{al}^{+}\end{array}$ & 2010 & GPCOG-It & $\begin{array}{l}\text { Cribado de demencia en la } \\
\text { práctica en general }\end{array}$ \\
\hline $\begin{array}{l}\text { Healey } \mathrm{AC}, \text { Rutledge CM, } \\
\text { Bluestein } \mathrm{D}^{+}\end{array}$ & 2011 & $\begin{array}{l}\text { ITAS (Insomnia Treatment } \\
\text { Acceptability Scale) }\end{array}$ & $\begin{array}{l}\text { Evaluar la adherencia al } \\
\text { tratamiento del insomnio }\end{array}$ \\
\hline $\begin{array}{l}\text { Gaynes BN, DeVeaugh- } \\
\text { Geiss J, et al }^{+}\end{array}$ & 2010 & M-3 checklist & $\begin{array}{l}\text { Trastorno depresivo, bipolar, } \\
\text { estrés postraumático }\end{array}$ \\
\hline $\begin{array}{l}\text { Campbell-Sills L, Norman } \\
\text { SB, Craske MG, et } \mathrm{al}^{+}\end{array}$ & 2009 & $\begin{array}{l}\text { OASIS (Overall Anxiety Severity } \\
\text { and Impairment Scale) }\end{array}$ & $\begin{array}{l}\text { Deterioro relacionado con la } \\
\text { ansiedad }\end{array}$ \\
\hline 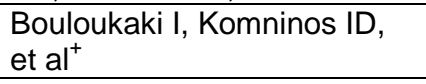 & 2013 & $\begin{array}{l}\text { OSAS (Obstructive Sleep Apnoea } \\
\text { Syndrome) }\end{array}$ & $\begin{array}{l}\text { Identificar pacientes con trastornos } \\
\text { del sueño }\end{array}$ \\
\hline $\begin{array}{l}\text { Mohr DC, Ho J, Duffecy J, } \\
\text { Baron KG, et al }{ }^{++}\end{array}$ & 2010 & $\begin{array}{l}\text { PBPT (Perceived Barriers to } \\
\text { Psychological Treatment scales) }\end{array}$ & $\begin{array}{l}\text { Evaluar las barreras percibidas } \\
\text { para el tratamiento psicológico }\end{array}$ \\
\hline $\begin{array}{l}\text { French P, Owens J, Parker } \\
\mathrm{S} \text {, et } \mathrm{al}^{+}\end{array}$ & 2012 & $\begin{array}{l}\text { PCCL (Early Detection Primary } \\
\text { Care Checklist) }\end{array}$ & $\begin{array}{l}\text { Identificar tempranamente los } \\
\text { estados psicóticos en jóvenes }\end{array}$ \\
\hline $\begin{array}{l}\text { Van Dam D, Ehring T, Vedel } \\
\text { E, et } \mathrm{al}^{+}\end{array}$ & 2010 & $\begin{array}{l}\text { PC-PTSD (Primary Care } \\
\text { Posttraumatic Stress Disorder } \\
\text { screening questionnaire) }\end{array}$ & $\begin{array}{l}\text { Evaluar el estrés postraumático en } \\
\text { pacientes con trastorno por uso de } \\
\text { sustancias }\end{array}$ \\
\hline $\begin{array}{l}\text { Queen } \mathrm{AH} \text {, Ehrenreich-May } \\
\text { J, el } \mathrm{al}^{+}\end{array}$ & 2012 & PD (Panic Disorder) & $\begin{array}{l}\text { Tamizaje para el desorden del } \\
\text { pánico en adolescentes }\end{array}$ \\
\hline $\begin{array}{l}\text { Patel V, Araya R, } \\
\text { Chowdhary N, et al }\end{array}$ & 2008 & $\begin{array}{l}\text { PHQ (Primary Health } \\
\text { Questionnaire) } \\
\text { GHQ (General Health } \\
\text { Questionnaire) } \\
\text { SRQ (Self-Reporting } \\
\text { Questionnaire) } \\
\text { K10 (Kessler Psychological } \\
\text { Distress Scale) } \\
\text { K6 (Kessler Psychological Distress } \\
\text { Scale) }\end{array}$ & $\begin{array}{l}\text { Comparar desordenes comunes } \\
\text { en salud mental }\end{array}$ \\
\hline $\begin{array}{l}\text { Arroll B, Goodyear-Smith F, } \\
\text { Crengle S, et } \mathrm{al}^{+}\end{array}$ & 2010 & $\begin{array}{l}\text { PHQ-9 } \\
\text { PHQ-2 (Patient Health } \\
\text { Questionnaire) }\end{array}$ & Depresión mayor \\
\hline $\begin{array}{l}\text { Allgaier AK, Pietsch K, Frühe } \\
\mathrm{B} \text {, et } \mathrm{al}^{+}\end{array}$ & 2012 & $\begin{array}{l}\text { PHQ-9 (Patient Health } \\
\text { Questionnaire) } \\
\text { PHQ-2 }\end{array}$ & $\begin{array}{l}\text { Instrumento de cribado para la } \\
\text { depresión en adolescentes }\end{array}$ \\
\hline
\end{tabular}




\begin{tabular}{|c|c|c|c|}
\hline $\begin{array}{l}\text { Zuithoff NP, Vergouwe } \mathrm{Y} \text {, } \\
\text { King M, et } \mathrm{al}^{+}\end{array}$ & 2010 & $\begin{array}{l}\text { PHQ-9 (Patient Health } \\
\text { Questionnaire) } \\
\text { PHQ-2 }\end{array}$ & $\begin{array}{l}\text { Detectar trastorno depresivo } \\
\text { mayor en APS }\end{array}$ \\
\hline 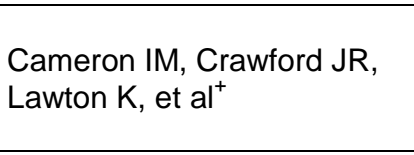 & 2008 & $\begin{array}{l}\text { PHQ-9 (Patient Health } \\
\text { Questionnaire) } \\
\text { HADS-D (Hospital Anxiety and } \\
\text { Depression Scale) }\end{array}$ & Evaluar la depression \\
\hline $\begin{array}{l}\text { Yazici Güleç M, Güleç H, } \\
\text { Simşek G, et } \text { al }^{+}\end{array}$ & 2012 & $\begin{array}{l}\text { PHQ-SADS (Patient Health } \\
\text { Questionnaire-Somatic, Anxiety, } \\
\text { and Depressive Symptoms) }\end{array}$ & $\begin{array}{l}\text { Evaluar la ansiedad somática y } \\
\text { síntomas depresivos }\end{array}$ \\
\hline $\begin{array}{l}\text { Magnil M, Gunnarsson R, } \\
\text { Bjørkelund } \mathrm{C}^{++}\end{array}$ & 2011 & $\begin{array}{l}\text { PRIME-MD } \\
\text { MADRS-S }\end{array}$ & $\begin{array}{l}\text { Detectar síntomas depresivos en } \\
\text { ancianos }\end{array}$ \\
\hline $\begin{array}{l}\text { Boustani M, Perkins AJ, } \\
\text { Monahan } \mathrm{P}, \text { et }^{+}{ }^{+}\end{array}$ & 2008 & PRISM-PC questionnaire & Detectar demencia \\
\hline Chiu JF, Chokka PR ${ }^{++}$ & 2011 & $\begin{array}{l}\text { ProBiD-PC (Bipolar Disorder } \\
\text { symptoms in Primary Care) }\end{array}$ & $\begin{array}{l}\text { Prevalencia del trastorno bipolar } \\
\text { en APS }\end{array}$ \\
\hline $\begin{array}{l}\text { Bellón JÁ, de Dios Luna J, } \\
\text { King } \mathrm{M} \text {, et } \mathrm{al}^{+}\end{array}$ & 2011 & $\begin{array}{l}\text { PSRA (PredictD-Spain risk } \\
\text { algorithm) }\end{array}$ & $\begin{array}{l}\text { Trastorno de pánico en el } \\
\text { adolescente }\end{array}$ \\
\hline Perry M, Drasković I, et al ${ }^{+}$ & 2010 & Qis (Quality indicators) & $\begin{array}{l}\text { Diagnostico y manejo de la } \\
\text { demencia }\end{array}$ \\
\hline $\begin{array}{l}\text { Luciano JV, Bertsch J, } \\
\text { Salvador-Carulla L, et } \mathrm{al}^{+}\end{array}$ & 2010 & SDS (Sheehan Disability Scale) & $\begin{array}{l}\text { Escala la discapacidad por } \\
\text { depresión mayor o trastorno de } \\
\text { pánico }\end{array}$ \\
\hline 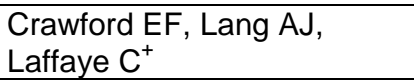 & 2008 & TEQ & Experiencias traumaticas \\
\hline $\begin{array}{l}\text { Mavaddat N, Lester HE, Tait } \\
\mathrm{L}^{+}\end{array}$ & 2009 & $\begin{array}{l}\text { The Patient Experience } \\
\text { Questionnaire }\end{array}$ & $\begin{array}{l}\text { Cuestionario de pacientes en salud } \\
\text { mental }\end{array}$ \\
\hline $\begin{array}{l}\text { Bellón JA, Moreno-Küstner } \\
\text { B, Torres-González F, et al }\end{array}$ & 2008 & The predictD-Spain study & $\begin{array}{l}\text { Predecir la aparición de depresión } \\
\text { mayor en APS }\end{array}$ \\
\hline 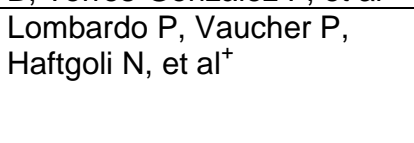 & 2011 & Three-question screening test & $\begin{array}{l}\text { Depresión mayor con preguntas de } \\
\text { ayuda en pacientes con alguna } \\
\text { dolencia física }\end{array}$ \\
\hline $\begin{array}{l}\text { Mohd-Sidik S, Arroll B, } \\
\text { Goodyear-Smith F, et al }\end{array}$ & 2011 & $\begin{array}{l}\text { TQWHQ (Two Questions With } \\
\text { Help Question) }\end{array}$ & $\begin{array}{l}\text { Preguntas para evaluar la } \\
\text { depresión }\end{array}$ \\
\hline Chin WY, Lam C, Wong $\mathrm{C}^{+}$ & 2011 & $\begin{array}{l}\text { Attitudes to Psychological } \\
\text { Interventions and Counseling in } \\
\text { Primary Care (APIC-PC) survey }\end{array}$ & $\begin{array}{l}\text { Conocimientos y actitudes frente a } \\
\text { intervenciones psicológicas }\end{array}$ \\
\hline
\end{tabular}

${ }^{+}$Base de datos de la que se obtuvo el estudio: Pubmed, ${ }^{++}$Base de datos de la que se obtuvo el estudio: Embase

Tabla VII: Instrumentos para evaluar otras áreas temáticas $\mathbf{n = 2 8}$

\begin{tabular}{|c|c|c|c|}
\hline Autores & Año & Instrumento & $\begin{array}{l}\text { Área de evaluación - Objetivo del } \\
\text { Instrumento }\end{array}$ \\
\hline $\begin{array}{l}\text { Aabenhus } \mathrm{R} \text {, Thorsen } \mathrm{H} \text {, } \\
\text { Siersma V, et al }\end{array}$ & 2013 & ARTIQ & Infecciones del tracto respiratorio \\
\hline $\begin{array}{l}\text { Kaarne T, Aalto M, } \\
\text { Kuokkanen M, et al }\end{array}$ & 2010 & AUDIT-C AUDIT-3, AUDIT-QF & Pacientes en salud ocupacional \\
\hline $\begin{array}{l}\text { Hallegraeff JM, van der } \\
\text { Schans CP, Krijnen WP, et } \\
\mathrm{al}^{+}\end{array}$ & 2013 & $\begin{array}{l}\text { Brief Illness Perception } \\
\text { Questionnaire }\end{array}$ & $\begin{array}{l}\text { Enfermedad en paciente con dolor } \\
\text { agudo no especifico de espalda baja }\end{array}$ \\
\hline $\begin{array}{l}\text { Rebollo P, Castejón I, } \\
\text { Cuervo J, et al }\end{array}$ & 2010 & $\begin{array}{l}\text { CAT based on Item Response } \\
\text { Theory (IRT) }\end{array}$ & $\begin{array}{l}\text { Calidad de vida relacionada con la } \\
\text { salud }\end{array}$ \\
\hline $\begin{array}{l}\text { Puig Ribera A, Peña } \\
\text { Chimenis O, Romaguera } \\
\text { Bosch } \mathrm{M} \text {, et } \mathrm{al}^{+}\end{array}$ & 2012 & CBPAAT-EBPAAT & Identificar la inactividad física \\
\hline $\begin{array}{l}\text { Block M, Ehrenworth JF, } \\
\text { Cuce VM, et al }\end{array}$ & 2013 & $\begin{array}{l}\text { CHEQ (Coordination of Handoff } \\
\text { Effectiveness Questionnaire) }\end{array}$ & $\begin{array}{l}\text { Escala de clima del trabajo en } \\
\text { equipo }\end{array}$ \\
\hline $\begin{array}{l}\text { Brito-Brito PR, Rodríguez- } \\
\text { Álvarez C, Sierra-López A, } \\
\text { et al }{ }^{+}\end{array}$ & 2012 & CVRS & $\begin{array}{l}\text { Evaluar la calidad de vida de los } \\
\text { pacientes }\end{array}$ \\
\hline $\begin{array}{l}\text { Bernhardsson S, Larsson } \\
\mathrm{ME}^{++}\end{array}$ & 2013 & $\begin{array}{l}\text { EBP and guidelines in physical } \\
\text { therapy }\end{array}$ & $\begin{array}{l}\text { Medir las actitudes, conocimientos, } \\
\text { comportamientos, requisitos y } \\
\text { barreras relacionadas con la practica } \\
\text { basada en la evidencia y directrices } \\
\text { en la terapia física }\end{array}$ \\
\hline
\end{tabular}




\begin{tabular}{|c|c|c|c|}
\hline 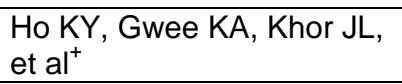 & 2008 & GERD (Gastroesophageal Reflux) & $\begin{array}{l}\text { Identificar población con reflujo } \\
\text { gastroesofágico }\end{array}$ \\
\hline 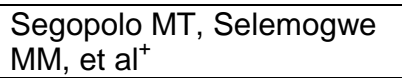 & 2009 & $\begin{array}{l}\text { GHQ-28 (General Health } \\
\text { Questionnaire) }\end{array}$ & Evaluar la salud general \\
\hline $\begin{array}{l}\text { De la Revilla L, de los Ríos } \\
\text { Álvarez AM, Luna del } \\
\text { Castillo Jde D, et al }{ }^{+}\end{array}$ & 2011 & MG (Migratory grief) & $\begin{array}{l}\text { Dolor en población inmigrante y } \\
\text { relación con variables } \\
\text { sociodemográficas }\end{array}$ \\
\hline $\begin{array}{l}\text { Cabrero-García J, Ramos- } \\
\text { Pichardo JD, Muñoz- } \\
\text { Mendoza CL, et } \mathrm{al}^{+} \\
\end{array}$ & 2012 & Mobility item bank & $\begin{array}{l}\text { Evaluar movilidad de adultos } \\
\text { mayores }\end{array}$ \\
\hline Láinez MJ, Castillo J, et $\mathrm{al}^{+}$ & 2010 & $\begin{array}{l}\text { MS-Q (Migraine Screen } \\
\text { Questionnaire) }\end{array}$ & Detección de migraña \\
\hline $\begin{array}{l}\text { Linton SJ, Nicholas M, } \\
\text { MacDonald S }\end{array}$ & 2011 & $\begin{array}{l}\text { OMPSQ (Örebro Musculoskeletal } \\
\text { Pain Screening Questionnaire) }\end{array}$ & Dolor musculoesqueletico \\
\hline Frilund M, Fagerström L+ & 2009 & OPC instrument & Cuidado en el envejecimiento \\
\hline $\begin{array}{l}\text { Georgy EE, Carr EC, Breen } \\
\mathrm{AC}^{+}\end{array}$ & 2013 & $\begin{array}{l}\text { Patients and Doctors } \\
\text { Expectations Questionnaire }\end{array}$ & Manejo del dolor de espalda \\
\hline $\begin{array}{l}\text { Perrot S, Poiraudeau S, } \\
\text { Kabir M, et al }\end{array}$ & 2008 & PCI (Pain Coping Inventory) & $\begin{array}{l}\text { Afrontamiento de dolor en pacientes } \\
\text { con artrosis de cadera y rodilla }\end{array}$ \\
\hline $\begin{array}{l}\text { Van Empel IW, Aarts JW, } \\
\text { Cohlen BJ, et al }^{+}\end{array}$ & 2010 & $\begin{array}{l}\text { PCQ-infertility (Patient- } \\
\text { centredness questionnaire- } \\
\text { infertility) }\end{array}$ & Cuidado de la fertilidad e infertilidad \\
\hline $\begin{array}{l}\text { Thomas TL, Strickland OL, } \\
\text { DiClemente R, et al }^{+}\end{array}$ & 2013 & $\begin{array}{l}\text { PHPVS (Parental Human } \\
\text { Papillomavirus Vaccine Survey) }\end{array}$ & Tamizaje de VPH \\
\hline $\begin{array}{l}\text { Karekla M, Pilipenko N, } \\
\text { Feldman } \mathrm{J}^{+}\end{array}$ & 2012 & $\begin{array}{l}\text { PHQ (Patient Health } \\
\text { Questionnaire) }\end{array}$ & Salud del paciente \\
\hline $\begin{array}{l}\text { Van Ravesteijn } \mathrm{H} \text {, } \\
\text { Wittkampf K, Lucassen } \mathrm{P} \text {, } \\
\text { et } \mathrm{al}^{+}\end{array}$ & 2009 & $\begin{array}{l}\text { PHQ-15 (Patient Health } \\
\text { Questionnaire) }\end{array}$ & Somatotipos \\
\hline $\begin{array}{l}\text { Halcomb EJ, Davidson PM, } \\
\text { Caldwell B, et al }^{+}\end{array}$ & 2010 & $\begin{array}{l}\text { PPE (Professional Practice } \\
\text { Environment Scale) }\end{array}$ & Salud ocupacional \\
\hline $\begin{array}{l}\text { Heneweer } \mathrm{H} \text {, van } \\
\text { Woudenberg NJ, van } \\
\text { Genderen } \mathrm{F} \text {, et } \mathrm{al}^{+}\end{array}$ & 2010 & $\begin{array}{l}\text { Questionnaire-Dutch Language } \\
\text { Version }\end{array}$ & Dolor agudo de espalda \\
\hline $\begin{array}{l}\text { Pace F, Scarlata P, Casini } \\
V \text {, et } \mathrm{al}^{+}\end{array}$ & 2008 & $\mathrm{RDQ}$ & Reflujo gastroesofagico \\
\hline $\begin{array}{l}\text { Fernández Cabrera T, } \\
\text { Medina Anzano S, Herrera } \\
\text { Sánchez IM, et al }{ }^{+}\end{array}$ & 2011 & self-efficacy scale & $\begin{array}{l}\text { Autoeficacia de adultos en la } \\
\text { actividad física regular }\end{array}$ \\
\hline Bentur N, King $\mathrm{Y}^{+}$ & 2010 & SF-12 & $\begin{array}{l}\text { Calidad de vida en personas } \\
\text { mayores residentes en la comunidad }\end{array}$ \\
\hline Romero Ortuño $\mathrm{R}^{+}$ & 2011 & $\begin{array}{l}\text { SHARE-FI (The Frailty Instrument } \\
\text { for primary care of the Survey of } \\
\text { Health, Ageing and Retirement in } \\
\text { Europe) }\end{array}$ & Salud, envejecimiento y jubilación \\
\hline $\begin{array}{l}\text { Cramm JM, Strating MM, } \\
\text { de Vreede PL, et } \mathrm{al}^{+}\end{array}$ & 2012 & SMAS & Evaluar el alta de pacientes mayores \\
\hline
\end{tabular}

\section{DISCUSIÓN}

En esta revisión se incluyeron 128 artículos que cumplieron con los criterios de inclusión; se encontraron diferentes tipos de instrumentos validados los cuales se enmarcaron dentro de la aplicación de la estrategia de APS. Las categorías obtenidas posterior al análisis fueron Estilos de vida y enfermedades crónicas, Estrategia de APS - Administración, Estrategia de APS - Relación Equipo de salud Usuario, Salud Mental y Otras áreas temáticas. Los resultados obtenidos evidencian una alta heterogeneidad en las áreas temáticas que evalúan los instrumentos relacionados con la APS, y poco se centran en medir el grado en que la esta cumple sus funciones ${ }^{(14)}$. Adicionalmente, se enfocan a la evaluación de programas específicos que se desarrollan en el marco de la estrategia de APS y patologías que se atienden, en 
concordancia con los resultados presentados de una investigación realizada en países de baja renta per cápita, en donde la mayoría de las evaluaciones se enfocaron a programas específicos como los materno-infantiles y a indicadores acotados de estructura y procesos, pero han carecido de una clara conceptualización acerca de las funciones de la APS y de una congruente medición de las mismas para valorar su grado de alcance o consecución ${ }^{(15-16)}$.

Resultados de otros estudios han evidenciado una tendencia en la investigación en APS centrado en problemas inespecíficos y en segundo y tercer lugar aspectos psicosociales y clínicos ${ }^{(12)}$. Dentro de esta variedad de abordajes de la estrategia, el $23,4 \%$ de los instrumentos revisados hacen referencia a artículos que la evalúan con relación al Equipo de salud y el Usuario, en este sentido en Canadá se realizó un estudio para identificar los instrumentos que evalúan los atributos de la APS desde la perspectiva del paciente, frente a lo cual se concluyó que es necesario ajustar las herramientas existentes y/o desarrollar nuevos instrumentos ${ }^{(17)}$. En la categoría Otras áreas temáticas, se incluyeron instrumentos dirigidos a evaluar la actividad física, los sistemas musculo esquelético, digestivo y nervioso; la salud ocupacional, la salud general, la salud sexual y reproductiva y el cuidado de la salud del adulto mayor, las cuales algunos estudios han evidenciado como actividades o servicios prestados en el marco de la APS ${ }^{(18-19)}$.

La categoría de menor porcentaje abordada en los instrumentos analizados fue la de Estrategia de APS - Administración (5,4\%), datos que coinciden con lo planteado por Cuba-Fuentes $\mathrm{M}$, et al, quienes afirman que en la evaluación se otorga una mayor importancia a aspectos como la disponibilidad de tiempo y servicios, accesibilidad, continuidad de la atención, información que reciben sobre sus problemas de salud o la relación médico - paciente, mientras que ponen menos énfasis en los aspectos administrativos $^{(20)}$. Al respecto es importante tener en cuenta que en esta categoría los instrumentos encontrados son más recientes lo que podría sugerir que hasta ahora se está retomando este aspecto como relevante en el funcionamiento de la estrategia aumentándose las publicaciones en este campo. Con relación a la producción científica de artículos que evidencian instrumentos validados por años, la tendencia evidenciada mostró un aumento entre el 2008 y 2011 de instrumentos en salud mental, estilos de vida y enfermedades crónicas, coherente con los resultados del trabajo desarrollado en España donde describieron un aumento en las publicaciones en APS entre 2008 y $2012^{(21)}$.

Los resultados encontrados denotan la necesidad de avanzar en procesos de adaptación transcultural de los instrumentos que ya existen para evaluar las diferentes áreas de la APS ya que unas de las dificultades del uso internacional de estos instrumentos es la magnitud de los cambios que suelen sufrir cuando son adaptados a diferentes contextos sin atender a recomendaciones sobre este tipo de procesos ${ }^{(22)}$. Además, a partir de estos resultados, se evidencia que hay dificultades en la estandarización de instrumentos que evalúen la APS, esta misma dificultad ha sido señalada en otro tipo de evaluaciones en el sector salud ${ }^{(23-24)}$.

\section{CONCLUSIONES}

Existe una amplia y variada disponibilidad de instrumentos genéricos y específicos que han sido validados en el contexto mundial para evaluar la estrategia de APS, sobre los cuales es posible reconstruir la mejor evidencia como herramienta de trabajo. A pesar de la importancia dada a la estrategia y del énfasis puesto en la 
necesidad de evaluarla, los instrumentos utilizados para este fin han sido heterogéneos, lo que pone de manifiesto la diversidad de intereses e investigadores en el área. Dicha heterogeneidad en las categorías que evalúan los instrumentos, plantea un desafío metodológico enfocado a la adaptación transcultural que se debe realizar previo a la aplicación de los instrumentos en contextos distintos a su origen y al entendimiento de las funciones o dimensiones que debería cumplir la APS. La tendencia en la producción de instrumentos por año refleja el interés en el contexto mundial de iniciar la evaluación de la estrategia y su administración, toda vez que es en el 2012 donde se evidencia el inicio de publicaciones de instrumentos que tengan relación con esta categoría de análisis. El aumento en el número de instrumentos publicados evidencia la necesidad de llevar a cabo revisiones sistemáticas periódicas de la literatura, en las que también se evalúe la calidad los instrumentos en función de sus propiedades métricas.

\section{REFERENCIAS BIBLIOGRÁFICAS}

1. Organización Panamericana de la Salud, Organización Mundial de la Salud. Renovación de la atención primaria de salud en la Américas: documento de posición de la Organización Panamericana de la Salud/Organización Mundial de la Salud (OPS/OMS) Washington, D.C.: Biblioteca Sede OPS; 2007.

2. Autor; 2013.

3. Pizzanelli M, Ponzo J, Buglioli M, Toledo A, Casinelli M, Gómez A. Validación de Primary Care Assessment Tool (PCAT) en Uruguay. Rev Med Urug. [Carta al Editor]. $2011 ; 27(3): 187-2$.

4. Navarro López V, Martín-Zurro A, Violán Fors C. Proyecto de Investigación: La Atención Primaria de Salud en España y sus comunidades autónomas. Barcelona: semfyc ediciones; 2009.

5. Macinko J, Almeida C, Klingelhoefer de Sá P. A rapid assessment methodology for the evaluation of primary care organization and performance in Brazil. Health Policy and Planning. [Search]. 2007;22:167-10.

6. Donabedian A. Aspects of Medical Care Administration: Specifying Requirements for Health Care. Cambridge: Harvard University Press; 1973.

7. Sibthorpe B, Gardner K. A Conceptual Framework for Performance Assessment in Primary Health Care. Aust J Prim Health. 2007;13(2):96-7.

8. Hogg W, Rowan M, Russell G, Geneau R, Muldoon L. Framework for primary care organizations: the importance of a structural domain. Int $\mathrm{J}$ Qual Health Care. 2008;20(5):308-5.

9. Campbell S, Roland M, Buetow S. Defining quality of care. Soc Sci Med. 2000;51(11):1611-25.

10. Ministerio de Sanidad y Consumo. Revisión externa y validación de instrumentos metodológicos para la lectura crítica y la síntesis de la evidencia científica. Madrid: Plan Nacional para el SNS del MSC. Servicio de Evaluación de Tecnologías Sanitarias del País Vasco (Osteba);2006. Report No.: OSTEBA № 2006/02.

11. Mokkink LB, Terwee CB, Patrick DL, Alonso J, Stratford PW, Knol DL, et al. Cosmin checklist manual. Amsterdam: University Medical Center 2012.

12. Carratalá-Munuera M, Orozco-Beltrán D, Gil-Guillen V, Navarro-Perez J, Quirce F, Merino $\mathrm{J}$, et al. Análisis Bibliométrico de la producción Científica Internacional sobre atención primaria. Atención Primaria. 2012;44(11):651-8.

13. Bernardo WM, Nobre MRC, Jatene FB. A prática clínica baseada em evidências: parte II-buscando as evidências em fontes de informação. Rev Assoc Med Bras. 2004;50(1):104-8. 
14. Berra S. El estudio de las funciones de la atención primaria de la salud: Adaptación e implementación de los cuestionarios PCAT en Argentina. 1era ed: CONICET; 2012.

15. Macinko J, Starfield B, Erinosho T. The impact of primary healthcare on population health in low- and middle-income countries. J Ambul Care Manage. 2009;32(2):15021.

16. Haggerty JL, Lévesque J-F, Hogg W, Wong S. The strength of primary care systems. BMJ: British Medical Journal. 2013;346.

17. Lévesque J-F, Haggerty J, Beninguissé G, Burge F, Gass D, Beaulieu M-D, et al. Mapping the coverage of attributes in validated instruments that evaluate primary healthcare from the patient perspective. BMC Family Practice. 2012;13(1):20.

18. Autor; 2014.

19. Feldman L, Vivas E, Lugli Z, Alviarez V, Pérez MG, S B. La satisfacción del paciente hospitalario: una propuesta de evaluación. Rev Calid Asist. 2007;22:133-40.

20. Cuba-Fuentes M, Jurado A, Estrella E. Evaluación del cumplimiento de los atributos de la Atención Primaria y el grado de satisfacción de los usuarios en un establecimiento de primer nivel de atención. Rev Med Hered. [Investigación ]. $2011 ; 22(1): 4-5$.

21. López-Torres Hidalgo J, Basora Gallisà J, Orozco Beltrán D, Bellón Saameño JÁ. Mapa bibliométrico de la investigación realizada en atención primaria en España durante el periodo 2008-2012. Atención Primaria. (0).

22. Berra S, Hauser L, Audisio Y, Mántaras J, Nicora V, Celestina de Oliveira MM, et al. Validez y fiabilidad de la versión argentina del PCAT-AE para evaluar la atención primaria de salud. Rev Panam Salud Publica. [Investigación Original]. 2013;33(1):309.

23. Atehortúa S, Ceballos M, Gaviria CF, Mejía A. Evaluación de la calidad metodológica de la literatura en evaluación económica en salud en Colombia: una revisión sistemática. Biomédica. 2013;33(4):615-30.

24. Pane S, Solans M, Gaite L, Serra-Sutton V, Estrada M, Rajmil L. Instrumentos de calidad de vida relacionada con la salud en la edad pediátrica. Revisión sistemática de la literatura: actualización Agència d'Avaluació de Tecnología i Recerca Mèdiques Barcelona. 2006.

Recibido: 20 de abril 2014; Aceptado 11 de julio 2014

ISSN 1695-6141

๑) COPYRIGHT Servicio de Publicaciones - Universidad de Murcia 